\title{
PARAFUSOS PEDICULARES: ESTRUTURAS ANATÔMICAS EM RISCO NO TRATAMENTO DA ESCOLIOSE IDIOPÁTICA
}

\author{
PEDICLE SCREWS: ANATOMIC STRUCTURES AT RISK IN IDIOPATHIC \\ SCOLIOSIS TREATMENT
}

\author{
TORNILLOS PEDICULARES: LAS ESTRUCTURAS ANATÓMICAS EN RIESGO \\ EN EL TRATAMIENTO DE ESCOLIOSIS IDIOPÁTICA
}

Herton Rodrigo Tavares Costa ${ }^{1}$, Carlos Fernando Pereira da Silva Herrero², Helton luiz Aparecido Defino ${ }^{3}$

\begin{abstract}
RESUMO
Objetivo: Avaliar a posição das estruturas anatômicas em risco durante a inserção de parafusos pediculares na coluna torácica e sua relação com a variação do ângulo de Cobb. Métodos: Os parâmetros estudados foram: a medida do ângulo de Cobb nas radiografias e a posição da medula espinhal, da cavidade pleural e aorta na ressonância nuclear magnética em relação a uma linha de 40mm criada para simular o parafuso pedicular nas cinco vértebras apicais. Resultados: A distância da aorta ao corpo vertebral e o ângulo de segurança do lado convexo apresentaram diferença estatística quando relacionados com a variação do ângulo de Cobb medido. Conclusão: Os resultados apresentados sugerem maior risco de lesão da artéria aorta com o aumento do ângulo de Cobb e aumento do risco na inserção de parafusos pediculares no lado convexo da curvatura, quando se considera o ângulo de segurança.
\end{abstract}

Descritores: Coluna vertebral; Escoliose; Imagem por ressonância magnética; Radiografia; Medula espinhal.

\begin{abstract}
Objective: To evaluate the position of anatomical structures at risk during insertion of pedicle screws in the thoracic spine and its relation to the variation of Cobb angle. Methods: The parameters studied were: the degree of Cobb angle on radiographs and the position of the spinal cord, the pleural cavity and aorta on MRI in relation to a line of $40 \mathrm{~mm}$ created to simulate the pedicle screw in the five apical vertebrae. Results: The distance from the aorta to the vertebral body and the angle of security of the convex side showed statistical difference when related to variation of Cobb angle measured. Conclusion: The results suggest a greater risk of injury to the aorta with increasing Cobb angle and increased risk in the insertion of pedicle screws on the convex side of curvature when considering the safety angle.
\end{abstract}

Keywords: Spine; Scoliosis; Magnetic resonance imaging; Radiography; Spinal cord.

RESUMEN

Objetivo: Evaluar la posición de las estructuras anatómicas en situación de riesgo durante la inserción de tornillos pediculares en la columna torácica y su relación con la variación del ángulo de Cobb. Métodos: Los parámetros estudiados fueron: la medida del ángulo de Cobb en las radiografías y la posición de la médula espinal, la cavidad pleural y la aorta en la RM en relación con una línea de 40mm, diseñada para simular el tornillo pedicular en el plano de las cinco vértebras apicales. Resultados: La distancia desde la aorta hacia el cuerpo vertebral y el ángulo de seguridad en el lado convexo mostraron diferencia estadística relacionada con la variación del ángulo de Cobb medido. Conclusión: Los resultados sugieren un mayor riesgo de daño a la aorta con el aumento del ángulo de Cobb e incremento del riesgo en la inserción de tornillos pediculares en el lado convexo cuando se considera el ángulo de seguridad.

Descriptores: Columna vertebral; Escoliosis, Imagen por resonancia magnética; Radiografía; Médula espinal.

\section{INTRODUÇÃO}

O sistema de fixação pedicular no segmento torácico da coluna vertebral tem sido utilizado para o tratamento cirúrgico de diversas patologias como tumores ${ }^{1,2}$, fraturas ${ }^{3-6}$, deformidades hipercifóti$\operatorname{cas}^{7,8}$ e escoliose idiopática do adolescente ${ }^{9-11}$. Quando comparada ao sistema de fixação por ganchos, o sistema de fixação pedicular apresentou maiores correções cirúrgicas, com segmentos de fixação menores e menor perda de correção ${ }^{11,12}$.

Apesar de atualmente ser muito difundida, a fixação da coluna torácica por meio de parafusos pediculares tem o potencial de evoluir com complicações graves. Alterações morfológicas como os pedículos estreitos e rodados e o deslocamento das demais estruturas pela rotação produzem uma menor zona de segurança em termos de lesão à medula espinhal, pleura e aorta torácica, na fixação por parafusos pediculares.

O objetivo deste estudo foi determinar a relação entre o parafuso pedicular utilizado no tratamento de pacientes com diagnóstico de escoliose idiopática do adolescente e a medula espinhal, pleura e aorta torácica e analisar o valor do ângulo de segurança para inserção do parafuso utilizando imagens obtidas por RNM.

\section{MATERIAIS E MÉTODOS}

O trabalho foi aprovado pelo comitê de ética em pesquisa de nosso serviço. Foram retrospectivamente avaliados os exames de imagem de 19 pacientes com o diagnóstico de escoliose idiopática do adolescente, incluindo as radiografias na incidência antero-

1. Médico Residente do Departamento de Medicina e Reabilitação do Aparelho Locomotor do Hospital das Clínicas da Faculdade de Medicina de Ribeirão Preto da USP - Ribeirão Preto, SP, Brasil. 2. Médico Assistente do Departamento de Medicina e Reabilitação do Aparelho Locomotor do Hospital das Clínicas da Faculdade de Medicina de Ribeirão Preto da USP - Ribeirão Preto, SP, Brasil.

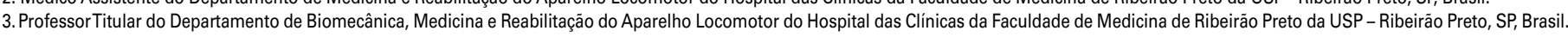

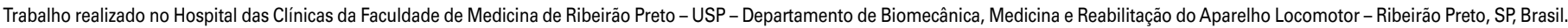

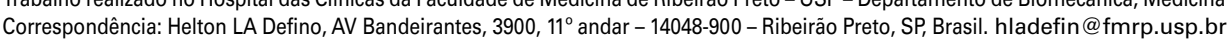

Recebido em 28/03/2012, aceito em 23/08/2012 
-posterior e a ressonância nuclear magnética da coluna vertebral.

A avaliação radiográfica compreendeu a medida do ângulo da curvatura por meio do método de Cobb na incidência antero-posterior da coluna vertebral.

Com relação ao exame de ressonância nuclear magnética (Philips Achieva 1,5T), foram analisados os cortes axiais das cinco vértebras apicais de cada paciente. As imagens axiais das vértebras de cada segmento torácico estudado foram transferidas para um programa de análise digital de imagens (eFilm Workstation versão 3). Foi analisada a posição anatômica dos pedículos torácicos em relação à medula espinhal medialmente e a cavidade pleural lateralmente em ambos os lados da curva. Utilizou-se uma linha de tamanho apropriado (40 mm) como simulador da inserção de um parafuso pedicular em cada vértebra. As linhas foram introduzidas de acordo com o ponto de entrada ideal descrito por Suk et al. $^{13}$ ao longo do eixo pedicular tanto no lado côncavo quanto no convexo das curvas.

Os parâmetros avaliados foram: após sua introdução no eixo pedicular, a linha correspondente ao parafuso foi deslocada medialmente até tocar a medula espinhal e lateralmente até tocar a cavidade pleural, e os valores angulares foram anotados. A distância do corpo vertebral à aorta torácica foi realizada através da medida da menor distância entre a parede externa da aorta e a cortical externa do corpo vertebral (Figura 1).

A análise estatística compreendeu o cálculo dos intervalos de confiança para as médias dos ângulos de segurança para inserção dos parafusos pediculares, da distância do corpo vertebral à aorta de cada vértebra e correlacionar estas medidas com a variação do ângulo de Cobb.

A ferramenta estatística utilizada foi o coeficiente de Spearman (Cs) que varia de -1 a 1. Valores próximos de 1 indicam alta correlação direta entre as variáveis evalores próximos de -1 indicam forte correlação inversa entre as variáveis. Os p-valores relacionados a eles são referentes ao teste de hipóteses, cuja hipótese nula é a de que o coeficiente é igual a zero.

Para se verificar se há diferença, em média, entre as vértebras, quanto ao ângulo e distância da aorta, foram ajustados modelos de efeitos mistos. Os modelos lineares de efeitos mistos (efeitos aleatórios e fixos) são utilizados na análise de dados onde as respostas de um mesmo indivíduo ou corpo de prova estão agrupadas e a suposição de independência entre observações num mesmo grupo não é adequada ${ }^{14,15}$. Esses modelos têm como pressuposto que seus resíduos tenham distribuição normal com média 0 e variância $\sigma 2$. Foi utilizado o procedimento PROC MIXED do software SAS versão 9.

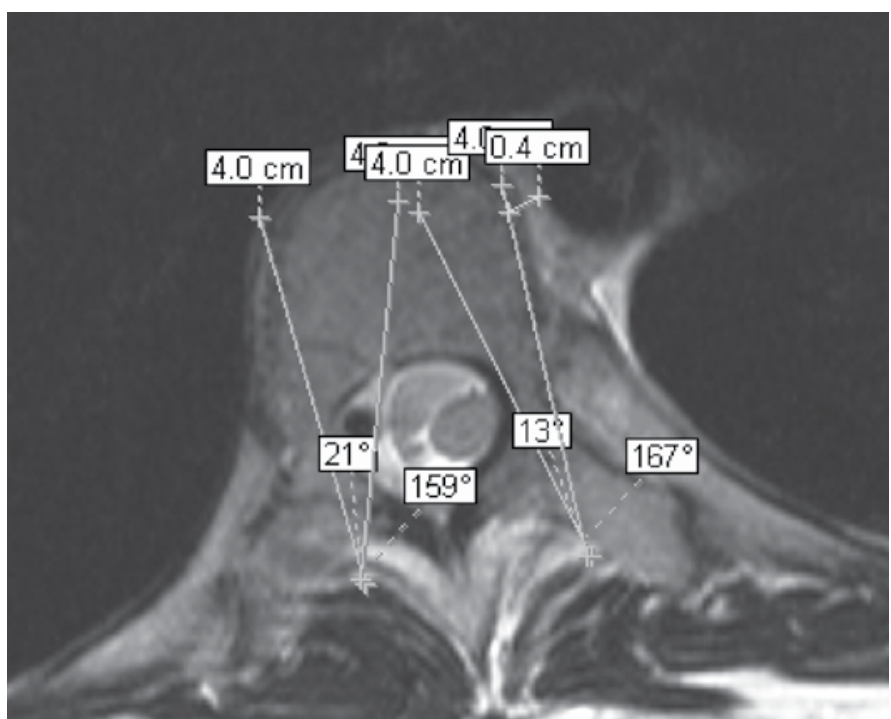

Figura 1. Corte axial de Ressonância Magnética ilustrando método de avaliação utilizada para demonstrar ângulo de segurança para inserção de parafusos pediculares e distância do corpo vertebral à aorta.

\section{RESULTADOS}

Foram avaliados os exames de 19 pacientes, sendo 18 do sexo feminino e um do sexo masculino. A idade variou de 11 a 25 anos $(15,73 \pm 3,95)$. O ângulo de Cobb variou de 29 a 96 graus $(60,93 \pm 20,06)$. Os dados gerais dos pacientes estão na Tabela 1 .

Um total de 95 vértebras (190 pedículos) foram analisadas, sendo a vértebra apical da deformidade, duas vértebras abaixo e duas vértebras acima. Os valores do ângulo de segurança para inserção dos parafusos pediculares da concavidade e convexidade estão ilustrados respectivamente nas Tabelas 2 e 3, e os valores da distância do corpo vertebral à aorta (Tabela 4).

Tabela 1. Dados gerais dos pacientes.

\begin{tabular}{cccc}
\hline Paciente & Sexo & $\begin{array}{c}\text { Idade } \\
\text { (anos) }\end{array}$ & $\begin{array}{c}\hat{A} \text { gulo } \\
\text { de Cobb } \\
\text { (graus) }\end{array}$ \\
\hline \hline 1 & Feminino & 15 & 56 \\
2 & Feminino & 14 & 80 \\
3 & Feminino & 11 & 60 \\
4 & Feminino & 13 & 33 \\
5 & Feminino & 15 & 40 \\
6 & Feminino & 13 & 50 \\
7 & Feminino & 14 & 44 \\
8 & Feminino & 18 & 42 \\
9 & Feminino & 21 & 75 \\
10 & Masculino & 24 & 90 \\
11 & Feminino & 25 & 68 \\
12 & Feminino & 17 & 83 \\
13 & Feminino & 14 & 80 \\
14 & Feminino & 11 & 76 \\
15 & Feminino & 12 & 54 \\
16 & Feminino & 14 & 29 \\
17 & Feminino & 18 & 42 \\
18 & Feminino & 15 & 96 \\
19 & Feminino & 15 & 54 \\
\hline
\end{tabular}

Tabela 2. Valores dos ângulos de segurança dos pedículos da concavidade (graus).

\begin{tabular}{cccccc}
\hline Paciente & $\begin{array}{c}\text { Vértebra } \\
\text { apical }\end{array}$ & $\begin{array}{c}\mathbf{1}^{\mathbf{a}} \\
\text { vertebra } \\
\text { abaixo }\end{array}$ & $\begin{array}{c}\mathbf{2}^{\mathbf{a}} \\
\text { vértebra } \\
\text { abaixo }\end{array}$ & $\begin{array}{c}\mathbf{1}^{\mathbf{a}} \\
\text { vertebra } \\
\text { acima }\end{array}$ & $\begin{array}{c}\mathbf{2}^{\mathbf{a}} \\
\text { vértebra } \\
\text { acima }\end{array}$ \\
\hline \hline 1 & 24 & 16 & 27 & 16 & 21 \\
2 & 43 & 31 & 29 & 19 & 36 \\
3 & 40 & 22 & 17 & 26 & 39 \\
4 & 16 & 13 & 16 & 15 & 28 \\
5 & 20 & 20 & 17 & 15 & 16 \\
6 & 17 & 33 & 30 & 23 & 17 \\
7 & 20 & 26 & 34 & 21 & 23 \\
8 & 15 & 21 & 25 & 17 & 29 \\
9 & 25 & 20 & 31 & 26 & 33 \\
10 & 30 & 45 & 55 & 29 & 22 \\
11 & 22 & 50 & 36 & 50 & 38 \\
12 & 29 & 33 & 30 & 29 & 28 \\
13 & 31 & 33 & 29 & 28 & 27 \\
14 & 33 & 27 & 21 & 37 & 29 \\
15 & 22 & 23 & 31 & 25 & 20 \\
16 & 15 & 16 & 31 & 25 & 21 \\
17 & 27 & 30 & 29 & 27 & 19 \\
18 & 16 & 18 & 27 & 11 & 33 \\
19 & 36 & 37 & 32 & 35 & 38 \\
\hline
\end{tabular}


Tabela 3. Valores dos ângulos de segurança para os pedículos da convexidade (graus).

\begin{tabular}{cccccc}
\hline Paciente & $\begin{array}{c}\text { Vértebra } \\
\text { apical }\end{array}$ & $\begin{array}{c}\mathbf{1}^{\mathbf{a}} \\
\text { vértebra } \\
\text { abaixo }\end{array}$ & $\begin{array}{c}\mathbf{2}^{\mathbf{a}} \\
\text { vértebra } \\
\text { abaixo }\end{array}$ & $\begin{array}{c}\mathbf{1}^{\mathbf{a}} \\
\text { vértebra } \\
\text { acima }\end{array}$ & $\begin{array}{c}\mathbf{2}^{\mathbf{a}} \\
\text { vértebra } \\
\text { acima }\end{array}$ \\
\hline \hline 1 & 25 & 25 & 22 & 22 & 21 \\
2 & 18 & 19 & 21 & 16 & 35 \\
3 & 29 & 29 & 34 & 22 & 22 \\
4 & 16 & 22 & 22 & 21 & 22 \\
5 & 26 & 23 & 35 & 20 & 26 \\
6 & 25 & 24 & 33 & 34 & 31 \\
7 & 31 & 28 & 35 & 28 & 30 \\
8 & 21 & 24 & 24 & 21 & 40 \\
9 & 30 & 25 & 33 & 30 & 25 \\
10 & 38 & 38 & 38 & 31 & 38 \\
11 & 43 & 41 & 47 & 27 & 34 \\
12 & 25 & 30 & 23 & 22 & 23 \\
13 & 39 & 41 & 43 & 42 & 38 \\
14 & 36 & 48 & 38 & 27 & 25 \\
15 & 35 & 38 & 38 & 32 & 34 \\
16 & 38 & 34 & 33 & 29 & 35 \\
17 & 34 & 30 & 29 & 38 & 38 \\
18 & 54 & 56 & 30 & 12 & 39 \\
19 & 27 & 27 & 36 & 23 & 33 \\
\hline
\end{tabular}

Tabela 4. Valores da distância corpo vertebral à aorta (cm).

\begin{tabular}{cccccc}
\hline Paciente & $\begin{array}{c}\text { Vértebra } \\
\text { apical }\end{array}$ & $\begin{array}{c}\mathbf{1}^{\mathbf{a}} \\
\text { vértebra } \\
\text { abaixo }\end{array}$ & $\begin{array}{c}\mathbf{2}^{\mathbf{a}} \\
\text { vértebra } \\
\text { abaixo }\end{array}$ & $\begin{array}{c}\mathbf{1}^{\mathbf{a}} \\
\text { vértebra } \\
\text { acima }\end{array}$ & $\begin{array}{c}\mathbf{2}^{\mathbf{a}} \\
\text { vértebra } \\
\text { acima }\end{array}$ \\
\hline \hline 2 & 1,6 & 0,8 & 1,1 & 1,4 & 1,3 \\
3 & 0,4 & 0,3 & 0,3 & 0,4 & 0,1 \\
4 & 0,4 & 0,5 & 0,5 & 0,4 & 0,3 \\
5 & 0,5 & 0,9 & 0,5 & 0,4 & 0,4 \\
6 & 0,2 & 0,3 & 0,2 & 0,5 & 0,2 \\
7 & 0,4 & 0,4 & 0,5 & 0,3 & 0,3 \\
8 & 0,5 & 0,3 & 0,3 & 0,2 & 0,2 \\
9 & 0,7 & 0,7 & 0,3 & 0,2 & 0,5 \\
10 & 0,4 & 0,2 & 0,1 & 0,3 & 0,5 \\
11 & 0,7 & 1,4 & 0,9 & 1 & 0,8 \\
12 & 0,8 & 0,4 & 0,3 & 0,5 & 0,4 \\
13 & 0,9 & 0,7 & 0,5 & 0,7 & 0,7 \\
14 & 0,8 & 0,4 & 0,7 & 0,7 & 0,6 \\
15 & 0,6 & 0,4 & 0,4 & 0,6 & 0,5 \\
16 & 1 & 0,6 & 0,5 & 0,3 & 0,8 \\
17 & 0,5 & 0,2 & 0,2 & 1,1 & 0,6 \\
18 & 0,3 & 0,3 & 0,2 & 0,3 & 0,2 \\
19 & 0,7 & 0,4 & 0,3 & 1,1 & 0,5 \\
\hline
\end{tabular}

Os valores das médias, desvio padrão e limites dos ângulos de segurança para a inserção dos parafusos nos pedículos em cada vértebra da concavidade e convexidade, e a distancia de cada corpo vertebral à aorta, estão demonstrados na Tabela 5. A média dos valores, o desvio padrão e os limites considerando todos os segmentos da convexidade e da concavidade estão representados na Tabela 6.

Correlacionando-se a média dos valores obtidos para o ângulo de Cobb com os ângulos de segurança, obteve-se diferença estatística significante apenas para as vértebras da convexidade $(p<0,05)$, mostrando que apenas os pedículos vertebrais deste lado apresentaram um menor ângulo de segurança em decorrência da maior deformidade da coluna, havendo assim maior risco de lesão à medula espinhal e pleura no lado da convexidade. Da mesma forma, para a aorta, obteve-se diferença estatística significante, mostrando que quanto maior o ângulo de Cobb, menor a distância do corpo vertebral à aorta e, portanto, maior será o risco de lesão (Tabela 7).
Tabela 5. Valores dos ângulos e distância do corpo vertebral à aorta, por vértebra. (* IC 95\% para a média).

\begin{tabular}{c|llcccc}
\hline & Vértebra & N & Média & $\begin{array}{c}\text { Desvio } \\
\text { Padrão }\end{array}$ & $\begin{array}{c}\text { Limite } \\
\text { inferior* }\end{array}$ & $\begin{array}{c}\text { Limite } \\
\text { Superior* }\end{array}$ \\
\hline \multirow{5}{*}{ Concavidade } & Apical & 19 & 25,32 & 8,52 & 21,41 & 29,23 \\
& $1^{\text {a } \text { abaixo }}$ & 19 & 27,05 & 9,95 & 22,48 & 31,62 \\
& $2^{\text {a } \text { abaixo }}$ & 19 & 28,79 & 8,55 & 24,87 & 32,71 \\
& $1^{\text {a }}$ acima & 19 & 24,95 & 9,17 & 20,74 & 29,16 \\
& $2^{\text {a } \text { acima }}$ & 19 & 27,21 & 7,44 & 23,8 & 30,62 \\
\hline \multirow{5}{*}{ Convexidade } & Apical & 19 & 31,05 & 9,23 & 26,81 & 35,29 \\
& $1^{\text {a } \text { abaixo }}$ & 19 & 31,68 & 9,72 & 27,22 & 36,14 \\
& $2^{\text {a } \text { abaixo }}$ & 19 & 32,32 & 7,35 & 28,95 & 35,69 \\
& $1^{\text {a }}$ acima & 19 & 26,16 & 7,43 & 22,75 & 29,57 \\
& $2^{\text {a } \text { acima }}$ & 19 & 31 & 6,54 & 28 & 34 \\
\hline \multirow{5}{*}{ Aorta } & Apical & 19 & 0,62 & 0,32 & 0,47 & 0,77 \\
& $1^{\text {a } \text { abaixo }}$ & 19 & 0,5 & 0,3 & 0,36 & 0,64 \\
& $2^{\text {a } \text { abaixo }}$ & 19 & 0,43 & 0,25 & 0,32 & 0,54 \\
& $1^{\text {a } \text { acima }}$ & 19 & 0,57 & 0,34 & 0,41 & 0,73 \\
& $2^{\text {a } \text { acima }}$ & 19 & 0,5 & 0,28 & 0,37 & 0,63 \\
\hline
\end{tabular}

Tabela 6. Descrição dos ângulos e distância do corpo vertebral à aorta, geral. ( ${ }^{*}$ IC 95\% para a média).

\begin{tabular}{l|ccccc}
\hline & $\begin{array}{c}\text { Número } \\
\text { de } \\
\text { vértebras }\end{array}$ & Média & $\begin{array}{c}\text { Desvio } \\
\text { Padrão }\end{array}$ & $\begin{array}{c}\text { Limite } \\
\text { inferior }\end{array}$ & $\begin{array}{c}\text { Limite } \\
\text { Superior }\end{array}$ \\
\hline Concavidade & 95 & $26,66^{\circ}$ & $8,69^{\circ}$ & $24,88^{\circ}$ & $28,44^{\circ}$ \\
Convexidade & 95 & $30,44^{\circ}$ & $8,27^{\circ}$ & $28,74^{\circ}$ & $32,14^{\circ}$ \\
Aorta & 95 & $0,53 \mathrm{~cm}$ & $0,3 \mathrm{~cm}$ & $0,47 \mathrm{~cm}$ & $0,59 \mathrm{~cm}$ \\
\hline
\end{tabular}

Tabela 7. Correlação dos ângulos de segurança com o valor do ângulo de Cobb.

\begin{tabular}{|c|c|c|c|}
\hline & Vértebra & p-valor & Diferenças \\
\hline Concavidade & $\begin{array}{c}\text { Apical } \\
1^{\mathrm{a}} \text { abaixo } \\
2^{\mathrm{a}} \text { abaixo } \\
1^{\mathrm{a}} \text { acima } \\
2^{\mathrm{a}} \text { acima }\end{array}$ & 0,43 & $\begin{array}{c}\text { *Não apresentou } \\
\text { diferença } \\
\text { estatística }\end{array}$ \\
\hline Convexidade & $\begin{array}{c}\text { Apical } \\
1^{\mathrm{a}} \text { abaixo } \\
2^{\mathrm{a}} \text { abaixo } \\
1^{\mathrm{a}} \text { acima } \\
2^{\mathrm{a}} \text { acima }\end{array}$ & 0,02 & $\begin{array}{c}\text { Apical, } 1^{\mathrm{a}} \text { abaixo, } \\
2^{\mathrm{a}} \text { abaixo, } 1^{\mathrm{a}} \\
\text { acima e } 2^{\mathrm{a}} \text { acima } \\
(\mathrm{p} \text {-valor }<0,05)\end{array}$ \\
\hline Aorta & $\begin{array}{c}\text { Apical } \\
1^{\mathrm{a}} \text { abaixo } \\
2^{\mathrm{a}} \text { abaixo } \\
1^{\mathrm{a}} \text { acima } \\
2^{\mathrm{a}} \text { acima }\end{array}$ & 0,03 & $\begin{array}{c}\text { Apical, } 1^{\mathrm{a}} \text { abaixo, } \\
2^{\mathrm{a}} \text { abaixo, } 1^{\mathrm{a}} \\
\text { acima e } 2^{\mathrm{a}} \text { acima } \\
(\mathrm{p}-\mathrm{val} \text { or }<0,05)\end{array}$ \\
\hline
\end{tabular}

\section{DISCUSSÃO}

A utilização de sistemas de fixação pedicular no tratamento de patologias da coluna vertebral é amplamente difundida ${ }^{16}$. Estudos biomecânicos, da taxa de artrodese, mobilização precoce e versatilidade evidenciaram superioridade da fixação pedicular em relação aos sistemas de fixação por meio de ganchos ou amarrilhos ${ }^{17,18}$.

Existe muita controvérsia ainda na utilização de parafusos pediculares no segmento alto e médio da coluna torácica, pois a complexa morfologia dos pedículos neste segmento da coluna vertebral pode implicar em aumento significativo do risco de estruturas anatômicas como a medula espinhal, cavidade pleural e artéria aorta ${ }^{10,19}$.

Estudos reportaram que o risco de inserção inadequada de um parafuso pedicular varia de $3 \%$ a $40 \%$, com 0 a $40 \%$ de complicações neurológicas atribuídas à instrumentação inapropriada ${ }^{11,20-23}$. No tratamento de deformidades da coluna vertebral, a utilização de parafusos pediculares no segmento torácico apresenta uma 
incidência relatada de mau posicionamento do parafuso pedicular que varia de $3 \%$ a 25\%, com complicações neurológicas relatadas em $0-0,9 \%$ dos $\operatorname{casos}^{11,20,24}$.

No intuito de diminuir o risco durante a inserção de parafusos pediculares ${ }^{25}$, foram desenvolvidos diversos métodos para aumentar a segurança do método, como fio guia intra-pediculares ${ }^{25}$, intensificador de imagens intra-operatório ${ }^{10,26}$, visualização direta da parede medial após laminectomia ${ }^{27}$, e sistemas de navegação guiados por imagens ${ }^{28}$

A penetração medial é certamente o risco mais temido durante a inserção de parafusos pediculares, pois pode levar à lesão da medula espinal. Estudos indicaram que o maior risco se encontra nos pedículos do lado côncavo da curvatura ${ }^{19,29}$. Porém, nosso estudo demonstrou que um ângulo de segurança para inserção de parafusos pediculares no lado convexo tem uma relação indireta com o ângulo de Cobb. Assim, com o aumento do ângulo de Cobb, o ângulo de segurança diminui nos pedículos do lado convexo e, conseqüentemente aumenta o risco de lesão neurológica.

Nos pacientes com escoliose, a aorta tem início aproximadamente na altura da quarta vértebra torácica, onde é anterior ao eixo médio do corpo vertebral. A diferença na posição é acentuada com curvas torácicas maiores, maior rotação dos segmentos apicais e hipocifose ${ }^{29}$. Enquanto estudos demonstraram que no segmento torácico médio a inserção de parafusos pediculares no lado côncavo possui uma margem de erro menor devido ao deslocamento posterolateral da aorta ${ }^{29,30}$, nosso estudo demonstrou que com o aumento do ângulo de Cobb a aorta se aproxima da cortical anterior dos corpos vertebrais no ápice da curva, aumentando o risco de lesão desta estrutura com a perfuração anterior do corpo vertebral.

\section{CONCLUSÃO}

A ressonância nuclear magnética se mostrou método eficaz na avaliação de estruturas anatômicas em risco com a inserção de parafusos pediculares utilizados no tratamento da escoliose idiopática do adolescente. Os resultados apresentados sugerem um maior risco de lesão da artéria aorta com o aumento do ângulo de Cobb e aumento do risco na inserção de parafusos pediculares no lado convexo da curvatura quando levado em consideração o ângulo de segurança.

\section{REFERÊNCIAS}

1. Amiot LP, Lang K, Putzier M, Zippel H, Labelle H. Comparative results between conventional and computer-assisted pedicle screw installation in the thoracic, lumbar, and sacral spine. Spine (Phila Pa 1976). 2000;25(5):606-14.

2. Jonsson B, Sjostrom L, Olerud C, Andreasson I, Bring J, Rauschning W. Outcome after limited posterior surgery for thoracic and lumbar spine metastases. Eur Spine J. 1996:5(1):36-44

3. Esses SI, Botsford DJ, Wright T, Bednar D, Bailey S. Operative treatment of spinal fractures with the AO internal fixator. Spine (Phila Pa 1976). 1991;16(Suppl 3):S146-S50.

4. Sasso RC, Cotler HB, Reuben JD. Posterior fixation of thoracic and lumbar spine fractures using DC plates and pedicle screws. Spine (Phila Pa 1976.). 1991;16(Suppl 3):S134-S9.

5. Sim E. Location of transpedicular screws for fixation of the lower thoracic and lumbar spine. Computed tomography of 45 fracture cases. Acta Orthop Scand. 1993:64(1):28-32.

6. Sjostrom L, Jacobsson $O$, Karlstrom G, Pech P, Rauschning W. CT analysis of pedicles and screw tracts after implant removal in thoracolumbar fractures. J Spinal Disord. 1993;6(3):225-31.

7. Chang KW. Oligosegmental correction of post-traumatic thoracolumbar angular kyphosis. Spine (Phila Pa 1976). 1993;18(13):1909-15.

8. Wu SS, Hwa SY, Lin LC, Pai WM, Chen PQ, Au MK. Management of rigid post-traumatic kyphosis. Spine (Phila Pa 1976). 1996;21(19):2260-6.

9. Delorme S, Labelle H, Aubin CE, de Guise JA, Rivard CH, Poitras B, et al. A three-dimensional radiographic comparison of Cotrel-Dubousset and Colorado instrumentations for the correction of idiopathic scoliosis. Spine (Phila Pa 1976). 2000;25(2):205-10.

10. Liljenqvist UR, Halm HF, Link TM. Pedicle screw instrumentation of the thoracic spine in idiopathic scoliosis. Spine (Phila Pa 1976). 1997;22(19):2239-45.

11. Suk SI, Lee CK, Kim WJ, Chung YJ, Park YB. Segmental pedicle screw fixation in the treatment of thoracic idiopathic scoliosis. Spine (Phila Pa 1976). 1995;20(12):1399-405

12. Suk SI, Lee CK, Min HJ, Cho KH, Oh JH. Comparison of Cotrel-Dubousset pedicle screws and hooks in the treatment of idiopathic scoliosis. Int Orthop. 1994;18(6):341-6.

13. Suk SI, Lee SM, Chung ER, Kim JH, Kim SS. Selective thoracic fusion with segmental pedicle screw fixation in the treatment of thoracic idiopathic scoliosis: more than 5-year follow-up. Spine (Phila Pa 1976). 2005;30(14):1602-9.

14. Littell RC, Henry PR, Ammerman CB. Statistical analysis of repeated measures data using SAS procedures. J Anim Sci. 1998;76(4):1216-31.

15. Schall R, Zucchini W. Model selection and the estimation of odds ratios in the presence of extraneous factors. Stat Med. 1990;9(10):1131-41.

16. Yahiro MA. Comprehensive literature review. Pedicle screw fixation devices. Spine (Phila Pa 1976). 1994;19(Suppl 20):2274S-8S.

17. Bess RS, Lenke LG, Bridwell KH, Cheh G, Mandel S, Sides B. Comparison of thoracic pedicle screw to hook instrumentation for the treatment of adult spinal deformity. Spine (Phila Pa 1976). 2007;32(5):555-61.

18. Liljenqvist $U$, Lepsien $U$, Hackenberg $L$, Niemeyer $T$, Halm $H$. Comparative analysis of pedicle screw and hook instrumentation in posterior correction and fusion of idiopathic thoracic scoliosis. Eur Spine J. 2002;11(4):336-43.

19. Cinotti G, Gumina S, Ripani M, Postacchini F. Pedicle instrumentation in the thoracic spine. A morphometric and cadaveric study for placement of screws. Spine (Phila Pa 1976). 1999;24(2):114-9.

20. Brown CA, Lenke LG, Bridwell KH, Geideman WM, Hasan SA, Blanke K. Complications of pediatric thoracolumbar and lumbar pedicle screws. Spine (Phila Pa 1976). 1998;23(14):1566-71.

21. Esses SI, Sachs BL, Dreyzin V. Complications associated with the technique of pedicle screw fixation. A selected survey of ABS members. Spine (Phila Pa 1976). 1993;18(15):2231-8.

22. Garfin SR. Spinal fusion: the use of bone screws in the vertebral pedicles. Summation. Spine (Phila Pa 1976). 1994;19(Suppl 20):2300S-5S.

23. Hirabayashi S, Kumano K, Kuroki T. Cotrel-Dubousset pedicle screw system for various spinal disorders. Merits and problems. Spine (Phila Pa 1976). 1991;16(11):1298-304.

24. Liljenqvist UR, Halm HF, LinkTM. Pedicle screw instrumentation of the thoracic spine in idiopathic scoliosis. Spine (Phila Pa 1976). 1997;22(19):2239-45.

25. Suk SI, Kim WJ, Lee SM, Kim JH, Chung ER. Thoracic pedicle screw fixation in spinal deformities: are they really safe? Spine (Phila Pa 1976). 2001;26(18):2049-57.

26. Gertzbein SD, Robbins SE. Accuracy of pedicular screw placement in vivo. Spine (Phila Pa 1976). 1990;15(1):11-4.

27. Frank EH. The use of small malleable endoscopes to assess pedicle screw placement: technical note. Minim Invasive Neurosurg. 1998;41(1):10-2.

28. Rampersaud YR, Simon DA, Foley KT. Accuracy requirements for image-guided spinal pedicle screw placement. Spine (Phila Pa 1976). 2001;26(4):352-9.

29. Sarlak AY, Buluc L, Sarisoy HT, Memisoglu K, Tosun B. Placement of pedicle screws in thoracic idiopathic scoliosis: a magnetic resonance imaging analysis of screw placement relative to structures at risk. Eur Spine J. 2008;17(5):657-62.

30. Sucato DJ, Duchene C. The position of the aorta relative to the spine: a comparison of patients with and without idiopathic scoliosis. J Bone Joint Surg Am. 2003;85(8):1461- 9 . 